\title{
The Property Driven Innovation of Materials for Use in Ophthalmology
}

\author{
by \\ L. Lawrence Chapoy \\ The High Performance Materials Company \\ 348 Ridge Road \\ Barrington Hills, IL 60010
}

\author{
and \\ John M. Lally \\ Advanced Medical Optics, Inc. \\ 1700 E. St. Andrew Place \\ Santa Ana, CA 97205
}

\begin{abstract}
$\underline{\text { Abstract }}$
Innovations are tracked and explained for four different classes of Ophthalmologic devices: contact lenses, intraocular lenses, intracorneal rings and viscoelastic agents. Successive improvements in the performance profile of these devices are driven by deficiencies that come to light for each version of the device, thus leading to a continuous evolution and product improvement. Standard considerations of materials engineering property profiles can and do apply. There is nothing mysterious about the use of such materials in connection with devices used for ophthalmology. The motivation leading to such innovation is the value proposition relating to the research and development expenditures and the promise of an eventual return.
\end{abstract}


Introduction: The entire history of ophthalmic materials and their respective devices goes back less than 100 years. Arguably the earliest ophthalmic material was glass used for very early contact lenses. Functional requirements of safety, efficacy and comfort drove the development of successive generations of materials. Some developments played off the old ideas and resulted in minor improvement. Other improvements were more radical and led to discontinuous improvements by utilizing a whole new materials engineering strategy.

Each new materials innovation was designed to overcome one or more deficiencies in the existing material. Each ophthalmic device application requires its own material(s) with a specific profile of engineering properties. In engineering terms, there is nothing particular about the fact that the material and device come into contact with the eye. Fundamental engineering will demystify any claims to the contrary.

Each type of ophthalmic device has undergone its own materials evolution improving performance and addressing specific materials related deficiencies for the application in question. The term performance as used here is taken in the broadest sense of the word:

- On eye performance relative to clinical metrics.

- Ease of manufacturing, yields and overall cost of production.

- Design features which are materials enabled.

Currently, we have arrived at a situation in which ophthalmic materials are integrated into highly engineered devices using all of the available sophistication of contemporary polymer technology. Since these devices are generally not subject to commodity pricing pressure, the use of specialty monomers and polymers can be permitted. That said, however, some materials innovations have been employed to improve quality standards, production yields, and production efficiencies in order to reduce costs. Market pressure to reduce costs derives from the need to stay competitive, as well as considerations of insurance re-imbursement, affordability and accessibility in the developing world.

Ophthalmic Materials: The use of ophthalmic materials has grown dramatically in recent years as creative treatments to various medical conditions have been devised through the use of corrective devices. Some classes of devices such as contact lenses have been in use for quite some time and have evolved through successive generations of innovation. Economically, contact lenses are by far the largest category and have thus received the most effort from the standpoint of research and development. Accordingly, one observes the largest and most complex evolutionary development for this category.

Other devices such as intracorneal rings, while of great significance to the individual patient, are of more recent vintage and are still in the secondary development phase. The size of the market for such specialized devices is quite small compared to that for contact lenses, and hence does not allow a great deal of latitude for materials research. Other device examples are in various stages of clinical development and are not in general use because of lack of regulatory approval. Materials obviously play a key role in defining device safety, but in efficacy as well.

The review presented here will give a snapshot of some applications of materials in ophthalmic devices and their respective evolution but is not intended to be an exhaustive review. The following device categories will be considered:

1. Contact Lenses

2. Intraocular Lenses

3. Intracorneal Rings

4. Viscoelastic Agents 
Since these are commercial products, the primary references will often be patents. In some cases, however, articles do appear later discussing the scientific and engineering merits rather than the legalistics of intellectual property protection.

\subsection{Contact Lenses}

1.1. Early History: Although Leonardo da Vinci is credited with originating the idea of a contact lens from a drawing dating 1508, the practical story really starts in 1887 in Wiesbaden, Germany. There. F.A. Muller, a maker of prosthetic eyes, at the suggestion of a physician Dr. Saemisch made a thin protective shell of clear blown glass to protect the remaining eye of a patient who had lost the other one some years earlier. The idea was to protect the remaining eye with the glass shell which the patient reportedly wore for some 20 years with vision intact and in "complete comfort." This was purely a protective device and in no way was there any attempt to incorporate usable optics into the device. This was probably the first example of what we refer to today as a "bandage lens." The stage was set, however, for what would emerge as a multi-billion dollar industry.

In 1888, Dr. A. Eugen Fick began experiments with refractive powered corneal contact lenses. These were the first corrective glass lenses to be worn. He asked Professor Ernst Abbe, an associate of Carl Zeiss to make these lenses by grinding them. Grinding was accompanied by almost $100 \%$ breakage and the blown lenses were of dubious precision. The lack of comfort led to rather short periods of wear. It is estimated that not more than 50 pairs were used before 1900. Furthermore, the aggressive nature of the lachrymal fluid attacked the glass giving it a useful lifetime of less than 18 months.

By 1912, it was reported that about 2000 lenses had been manufactured, mainly by Zeiss. During the 1920's probably not more than 5000 pairs were made both in Europe and the United States combined. The production grew steadily. From 1935 to 1939, at least 10,000 pairs of glass lenses were sold in the United States.

Alfred W. Mueller-Welt perfected the grinding and by the late 1930's had incorporated a system of stock lenses for the firm Mueller-Welt Brothers. The glass lenses suffered from manufacturing difficulties and lack of comfort. Presumably they were also very expensive at the time and were only for the privileged few.

1.2. Polymethylmethacrylate (PMMA) Contact Lenses: During the 1950's, Dr. MuellerWelt pioneered the development of plastic lenses made of PMMA. Lenses were injection molded initially, but were unsatisfactory due to the primitive nature of the process at that time. Lathe cutting operations were begun in about 1958. T. Obrig and I. Gyorrfy worked on this contemporaneously. There is some controversy as to who actually made the first plastic contact lens.

A hybrid lens consisting of central glass optics with a plastic scleral rim was also worked on by W.Feinbloom. Early on, better optics could be achieved with glass, but the plastic had other desirable properties. The concept of such a hybrid lens is still with us today, but current versions use more sophisticated materials for both the central portion and the scleral peripheral region: For example a rigid high Dk center portion with excellent optical properties promoting good eye health with good oxygen transmissibility can be surrounded by a soft hydrogel periphery for added comfort.

PMMA represented a significant advance relative to glass in its application to contact lenses in the following aspects:

- PMMA is a thermoplastic and hence was moldable by a variety of relatively inexpensive processes including compression molding from sheet stock, injection molding and polymerization casting from liquid polymer into a pre-form. 
- PMMA lenses can be lathe cut from preformed buttons.

- PMMA can be polished by mechanical and chemical processes.

- A finished PMMA lens can be easily modified to change the shape and power. Similarly, rejuvenation of worn lenses can easily be accomplished, e.g. cleaning, polishing, change of power, etc.

- Transparency is above $90 \%$ with no loss in time.

- PMMA rarely exhibits cracking or internal crazing.

- Index of refraction is 1.48

- Specific gravity is $1.19 \mathrm{~g} / \mathrm{cm}^{3}$ compared to $2.6 \mathrm{~g} / \mathrm{cm}^{3}$ for glass.

- PMMA has a relatively hard surface.

- PMMA is inert to lachrymal fluid.

- PMMA is easily and safely colored with dyes and/or pigment.

- Chemical cleaning and disinfection can be safely performed by the wearer.

- PMMA does not sustain bacterial growth.

Initially, PMMA had no chemical crosslinking so that its rigidity was not sufficiently high. The addition of chemical crosslinking solved that problem but also created other problems such as poor solvent resistance that led to crazing, cracking or even disappearance of the lens altogether! This is important for the cleaners, polishers and also accidental exposure to household chemicals, e.g. nail polish remover.

PMMA does have two severe limitations that it shares with glass that still needed to be overcome:

- Hard lenses of any kind are inherently more uncomfortable than soft lenses. In addition to the obvious commercial implications, comfort has been subjectively found to affect the quality of vision.

- Oxygen transmissibility [1] is crucial for a contact lens material. Oxygen to the cornea is supplied directly from the surrounding air. There are no blood vessels to feed the cornea with oxygen. Placing a barrier to oxygen over the cornea robs it of its supply of oxygen and this is not good for eye health. Corneal edema is the result and the response is lack of comfort. Wearers of these lenses feel the need to remove them after a lapse of hours depending upon the patient. Partial oxygen starvation occurs during sleeping with the closed eye lid being the barrier. Evolutionarily, the body is able to cope with this magnitude of reduction for Ca 8 hours. PMMA has almost no oxygen transmissibility. The only oxygen getting through is from seepage at the edges. Fenestration as well as various designs to promote tear mixing have been proposed with only a marginal benefit.

As it turned out, each of these shortcomings were addressed independently to produce two distinct categories of post PMMA lenses:

- Soft Hydrogel lenses

- Rigid Gas Permeable (RGP) lenses

1.3. Poly-t-butyl styrene lens: Around 1984, a lens was introduced based on this polymer. The idea was that the bulky t-butyl group would create extra free volume in the polymer in the glassy state. From the Free Volume model one would expect this to increase the diffusion coefficient of oxygen. The lens was never a big success, so evidently the clinical benefits in terms of comfort and edema were not big enough to have any practical impact. This was a commercial product from now defunct Wesley Jessen. 
1.4. Soft Hydrogel Lenses: The soft lens saga began in the early 1960's with Dr. Otto Wichterle a polymer chemist at the Czechoslovak Academy of Sciences in Prague who developed a hydrophilic plastic compound based on the monomer hydroxyethylmethacrylate (HEMA). This was truly a revolutionary step in the materials evolution for contact lenses. Wichterle was assisted by Dr. Drahoslav Lim who moved to the United States and ultimately became a professor at the University of California at San Diego.

There is considerable Cold War intrigue about the Communist regime's thirst for hard currency, their desire for the Czechs to show independence from the Soviets and the treatment of Dr. Wichterle after the Soviet invasion in 1968. Ultimately it was brought to Bausch and Lomb for commercialization.

1.4.1. The advantages of the HEMA based soft contact lenses were obvious:

- The soft lenses were much more comfortable than any of the hard lenses had been.

- The soft lens drapes over the cornea. Fewer prescriptions would be required. It was no longer necessary to manufacture the lenses on a made to order basis. Practitioners could have an inventory on hand permitting the immediate dispensing of lenses to patients.

- The poly-HEMA is a crosslinked hydrophilic plastic material. The water acts as a plasticizer. At its equilibrium water content it becomes soft but also allows for a finite transportation of oxygen from the surrounding air through the lens to the cornea. While this oxygen transport is still well below that of the closed eye it nonethe-less provides for considerably increased wearing time, perhaps as long as the work day, 8-10 hours.

- The poly-HEMA lenses lent themselves to high volume production, thus lowering the cost of manufacture and eventually leading to development of disposable contact lenses. Tear fluid deposit build-up on the lenses can thus be dealt with by taking a new one rather than dealing with cleaning of a fragile hydrogel contact lens. Since there is only a negligible amount of material in each lens, the cost is ultimately determined by the amount of automation employed and the volume produced.

These advantages resulted in rapid growth of the contact lens market after Bausch and Lomb received FDA approval in 1971.

1.4.2 Soft Lens Manufacturing: Soft hydrogel lens can be manufactured in a variety of ways with varying degrees of advantages and capital investment.

- Lathing: Since the dry poly-HEMA is a rigid plastic it can be lathed. HEMA can be polymerized in tubes to make rods of poly-HEMA. The rods can then be sliced into disks which can be cut on a lathe. Such lathes have evolved over the years from being cam-shaft driven to Computer Numerical Control (CNC) machines. This is reminiscent of the process used to make PMMA lenses.

- Spin Casting: Wichterle patented the so-called spin casting process in which the monomer is put into an open mold and spun while polymerized with UV light. The centrifugal force of the spinning makes the monomer climb the walls of the mold and produces a spherical cross section. Since the cornea of most patients tends to be spherical, the back surface of the lens needs no further treatment before it is placed on the eye. The radius of curvature is controlled by the spin speed. The front surface of the lens and its edges can be cut from the polymer on the bottom of the mold. This technology found its way to Bausch \& Lomb in connection with the deal with Professor Wichterle. 
- Single sided molding: A female mold with the base curve information on the bottom of the mold is charged with monomer and polymerized either thermally or with UV light. The front curve is then lathed. Only a few base curve parameters are required, eliminating the need for more expensive lathing operations. The mold materials require chemical resistance to the monomer mix. HEMA is an aggressive solvent so usually a crystalline polymer such as polypropylene is required. Since the final lens will be swollen with saline, the polymerized lens is made small to take into account the swelling from the hydration of the poly-HEMA.

- Double sided molding: A female mold is charged with monomer as above. A male mold is inserted into the female and polymerization is effected either thermally or with UV light. Since there are two mold halves the polymerized article contains both the front and back surface as well as the edges. No additional shaping is required. Adding an inert diluent to the monomer mix such as isopropanol produces lenses that do not undergo expansion when the saline replaces the diluent in a solvent exchange step. Adding water to the monomer mix directly adversely affects the polymerization chemistry.

- Using the identical formulation, molded lenses can have slightly different surface properties than lathed lenses since in the one case the lens surface is formed against a mold surface while in the other case the surface is essentially formed against itself in the rod. Given that logic, the molded lens surface should show at least some dependence on the choice of mold materials as well.

1.4.3 Post poly-HEMA hydrogels: Once the poly-HEMA picture became clear it opened the area to the development of a whole selection of suitable hyrdogels. By varying the water content, refractive index and other properties, new materials with unique characteristics were developed. The addition of acrylic acid enables one to increase the water content from the poly-HMA value of $38 \%$ to some higher value, ca 55\%. As the water content gets higher, however, the modulus of the lens goes down. To counteract this, the lenses are made thicker. The usual lens thickness is about 50-100 microns. Increasing the lens thickness much above 100 microns tends to make them uncomfortable for the patient. The refractive index of the lens which important for the optics is inversely proportional to water content. This tends to require a thicker lens as the refractive index goes down. The oxygen transport, $\mathrm{Dk}$, is dependent on the water content as shown in Figure 1, so the maximum amount of water which keeps the lens at a reasonable thickness, modulus and refractive index is a good compromise to obtain a lens with a reasonable overall property profile. 


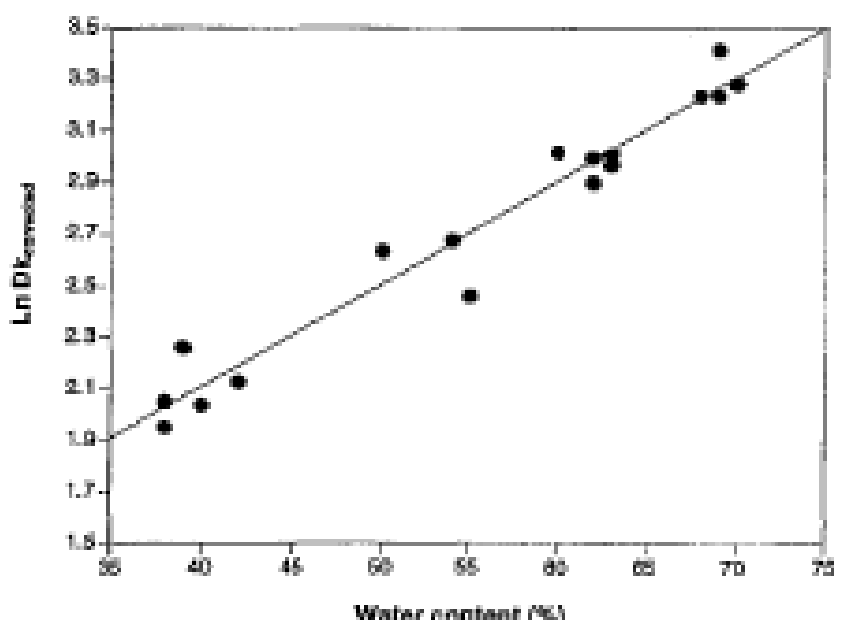

Figure 1. Correlation of Dk with percent water (log-linear plot). 1 barrer $=10 * *$ $11 \mathrm{~cm} 3$ ) $\mathrm{O} 2$ (at STP)/cm2s mmHg. The linear relationship is what would be expected from the applicability of the Free Volume Model. (P.B. Morgan and N. Efron, Contact Lens and Anterior Eye, 21,3 (1998).

The water is basically acting as a plasticizer for the rigid and the Free Volume Theory is working as it does for synthetic hydrophobic polymers. The WilliamsLandel-Ferry Theory should be applicable but it does not appear that it is well known to the ophthalmic community. The anionic nature of the resulting polymers led to the undesirable increased predisposition to tear fluid deposits. Lysozyme, one of the major tear components is positively charged.

There are many hydrophilic monomers to choose from. N-vinyl pyrolidone or acrylamide lead to non-ionic high water polymers. This reduces the predisposition to deposits. To decrease the water content to a manageable level, one can resort to using a co-monomer with the right co-polymerization parameters to avoid forming block copolymers which will cause opacity of the material. Acrylamide/methyl methacrylate copolymers were the basis of a commercial product, from now defunct Wesley Jessen.

A biomimetic hydrogel [2] has been proposed for use in contact lenses. The concept was that the increased biocompatibility would give a superior lens even at equal water content when compared to a synthetic hydrogel. The polymers were acrylates with phosphorylcholine based side chains. This should make them compatible with the lipid membranes in the corneal epithelium. Furthermore, since the polymer is zwitterionic, it should be less prone to attract charged proteins from the tear fluid. A commercial product was launched from now defunct contact lens division of Biocompatibles.

The use of functionalized macromers of poly-vinyl alcohol [3], PVA, represents a significant step forward in the desire to rationalize the manufacturing process and thereby lower costs. These cost improvements thus make it possible to provide a daily disposable lens to the consumer at a cost equivalent to the more traditional two week disposable hydrogels. Macromers are obtained from PVA oligomers which are functionalized with a pendant double bond. An aqueous solution of macromer of concentration appropriate to the water content of the final lens is subject to a photopolymerization in a contact lens mold. The chemistry involved is minimal because the polymer is already formed through the macromer. The photopolymerization serves only to form the hydrogel network. The number of moles of reaction is thus much less than when starting from monomer and the time of 
reaction is therefore much shorter. Furthermore, since the water is already present, no hydration step is required. Unlike monomers such as HEMA, the macromers are highly purified and not toxic and hence an extraction step is not required. Ciba Vision has a product based on this technology.

1.5. Silicone containing Lenses [4]: While the hydrogel lenses were being developed a completely different strategy was being pursued to make lenses that were able to transmit oxygen in order to promote eye health and improve wearing comfort. Sillicon containing polymers are known to have a high diffusion coefficient for oxygen.

1.5.1 Silicone rubber, poly-dimethylsiloxane is known to have oxygen transmissibility higher than pure water while being very soft and flexible. These materials are known for being very hydrophobic, so the properties could be expected to be totally different from the hydrogel materials While these lenses had some issues, their fatal flaw for on eye performance is what the clinicians referred to as "non-movement." For reasons that are not exactly clear these lenses tended to adhere to the cornea in some patients. There are components in the tear fluid that are thought to promote adhesion. Intensive development of a wide range of comonomers at Dow-Corning led to a material that alleviated the adhesion as well as making it more wettable and stronger than the original formulation. These lenses received FDA approval in the early 1980's. The lenses were not very comfortable and suffered from deposits from the tear fluids. However, the higher oxygen transmissibility did lead to improved eye health as predicted.

1.5.2. Silicone Rigid Gas Permeable (RGP) lenses: Other formulations based on silicone materials led to rigid lenses that still had the underlying advantage of high oxygen transmissibility. These lenses are based on silicone containing acrylates and methacrylates.

1.5.3. Fluoro-silicone RGP lenses [5]: While silicone containing polymers have a high diffusion, $\mathrm{D}$, coefficient for oxygen, the total oxygen flux, $\mathrm{Dk}$, is the product of the diffusion coefficient and the solubility of the oxygen, k. Fluorine is known to increase the solubility of oxygen. These polymers are similar to the silicone RGP materials but with some opportunistic substitution of fluorine. Standards have been set for $\mathrm{Dk} / \mathrm{t}$ where $\mathrm{t}$ is the thickness of the lens: 24 for edema free daily wear and 125 for edema free extended wear. Normal hydrogels currently in use are probably somewhat below the value of 24 . There are RGP lenses with reported values in excess of 150 . While the high $\mathrm{Dk} / \mathrm{t}$ values of these lenses would indicate good eye health, they are still not very comfortable because of their rigidity.

1.5.4. Fluorinated polyethers: These materials in the liquid state have been proposed as blood substitutes because of their excellent oxygen transporting properties. The fluorine content leads to very high oxygen solubility. At the same time, these materials have an extremely low glass transition temperature which gives them high gaseous diffusion constants at ambient temperature. This combination gives a very high value of Dk. Functionalized versions of the fluorinated polyethers can be prepared that will form networks under suitable conditions of polymerization. These networks form the basis for the rubbery contact lens materials. $3 \mathrm{M}$ pioneered work using this technology.

1.5.5. Surface Treatment: Since these silicone, fluoro-silicone and fluorolenses lenses all are very hydrophobic, surface treatments are required to improve comfort, biocompatibility and the predisposition to adhesion. These surface treatments can be accomplished by chemical baths, plasma treatments etc. A few nanometers of surface treatment is sufficient to alter the biocompatibility without effecting the bulk properties of the material. 
1.6 Silicone Hydrogels [6]: These materials combine the softness of hydrogels to provide for comfort with the high Dk values of RGP lenses to provide for eye health. Prepolymers of silicone containing units can be polymerized with hydrogel type monomers. The water contents of these materials are below that of conventional hydrogels. For these materials, an increase in the water content actually leads to a lowering of $\mathrm{Dk}$, while the modulus decreases. Although they were thought to be wettable because of the moderate water content, this has not proven to be true under clinical testing. A possible explanation for the non-wetting is the migration of silicone fragments to the hydrophobic air surface. Surface treatments are therefore still required. Non-charged lipid deposits are still a potential problem for the silicone hydrogels.

Since these two very different chemical compositions are not miscible, the process chemistry must control the two phase morphology in a very critical way. The domains of each phase cannot become large enough to scatter light and make the polymer useless as a lens material. At the same time a co-continuous network must be formed so that channels of silicone and hydrophilic containing polymer respectively extend from the front surface to the back surface in order to transport oxygen and ions. This is in effect a percolation system. Much has been written about percolation models and the discontinuity in transport phenomena that occurs when the concentration of one component becomes high enough to make a percolation path probable.

This development has had a very strong practical result for the contact lens market. In as much as these lenses seem to afford better eye health for little or no additional cost to the patient. The Dk of these lenses is greater than 100 in accordance with the requirents described above. Market share has jumped virtually from nil to $50 \%$ in less than 10 years. The poly-HEMA lens market share has gone down similarly. The product life cycle for the poly-HEMA lens will be about 40 years. This points out the need for continuing innovation and the fact that all products have a finite lifetime.

Ciba Vision (lotrafilcon A and lotrafilcon B) and Bausch \& Lomb (balafilcon A) pioneered these developments. Both companies use gas plasma technologies to create a hydrophilic wettable surface. Later, Vistakon commercialized a family of silicone hydrogel materials (galyfilcon A and senefilcon A) employing an "internal wetting agent" poly(vinyl pyrrolidone) to provide surface wettability instead of using a plasma treatment or separate coating step. More recently Menicon introduced a silicone hydrogel (asmofilcon A) with a plasma surface treatment. A new line of research initiated by Asahi Kasei Aime Co in Japan has given rise to a new silicone hydrogel (comfilcon A) marketed by Cooper Vision that combines a high equilibrium water content with a relatively high oxygen permeability while not requiring a separate surface treatment or internal wetting agent. Finally, Ciba Vision has recently introduced a high-precision lathecut silicone hydrogel lens (sifilcon A) which is plasma coated. Unlike all previously mentioned silicone hydrogel lenses which are all cast molded, this is a custom or made-toorder lens for patients with high refractive errors and aphakia. [7]

1.7 UV filtering Contact Lenses: Since contact lens wearers are less prone to wear sunglasses during sunny periods in the appropriate latitudes their eyes are subjected to greater doses of UV radiation than spectacle wearers. Sunglasses generally contain UV blocking additives. UV radiation damage is thought to be the cause of a number of eye complications one of which is cataract formation. It would appear therefore prudent to include some type of UV filtering additives into contact lens polymers. For rigid lenses made from PMMA and RGP materials traditional UV filtering additives such as benzophenones and benzotriazoles can be formulated into the polymer without fear of them being leached out. Hydrogels and soft materials in general with higher diffusion 
constants run the risk of having these additives leached out during the product life cycle. It has thus become critical to functionalize these additives onto acrylic moieties so they can be co-polymerized into the polymeric lens material. [8]

1.8 Colored contact lenses: Hydrogel contact lenses require the addition of a so-called handling or visibility tint so they don't disappear when they fall into the sink and can be located in a lens storage/disinfecting cleaning container. This can be achieved either by the introduction of a minute amount of non-leachable pigment or reaction with a reactive dye that will couple with reactive $-\mathrm{OH}$ groups on the polymer. A small amount of dye gives rise to the tint. As the tint becomes more intense it can enhance the natural color of the wearer's iris. The reactive dye can be imbibed into the finished lens together with an activator and reacted. The lenses can then be extracted to remove un reacted dye and residual activation material.

Cosmetic contact lenses are also articles of commerce. These can either be for prosthetic lenses for diseased or damaged eyes or lenses which make a cosmetic and fashion statement like lip stick and nail polish. These lenses can change the color of the wearer's iris. This can be achieved in a number of ways, one of which is to print the color on to the lens body. The strategy behind such a printing ink is as follows: For printing on a poly-HEMA lens the ink would consist of a low molecular weight poly-HEMA binder which was dissolved in HEMA monomer. To this solution would be added a colored pigment and polymerization initiator. After printing, the ink would be cured in an oven. The HEMA solvent would swell the surface of the lens body and polymerization would form an interpenetrating network, IPN, at the surfaces. This IPN would improve the adhesion between the printed layer and the lens body. Now defunct Wesley Jessen pioneered this technology [9].

1.9Corneal Inlays \& Onlays [10] : Lasik, a standard refractive surgery procedure involves the cutting of a thin flap of cornea using a microketatome. The exposed cornea is then sculpted by laser ablation to correct for refractive errors. The flap is then replaced so that the healing process is rapidly accelerated. An Inlay or Onlay is in essence a contact lens which is implanted under the flap to correct for refractive error without necessitating any removal of corneal tissue. The Onlay is implanted under a very shallow flap just under the epithelium. It should be removable and truly reversible since there is no stromal incision. The Inlay is implanted under a normal Lasik flap of $c a$ 50-100 microns. It is also easily removable since the flap never really re-integrates into the tissue and is almost fully reversible since there is no abrasion of corneal tissue. While some work has focused on adapting existing hydrogel materials already proven for contact lens wear, namely HEMAbased hydrogels, most success has resulted from the use of porous perfluoropolyether polymers which appear to satisfy the key requirements of biostability, biocompatibility, optical transparency and optimal nutrient flux. Since these are implants they are subject to an elevated regimen of clinical requirements and regulatory scrutiny than contact lenses, especially long term stability. As of this writing, these devices are in late stage clinical development.

1.10 Contact Lenses as a Vehicle for Controlled Release [11]: Contact lenses have long been viewed as an ideal vehicle for dosing medications or other agents into the eye. There are numerous advantages to this aside from convenience. The active molecule is allowed to act locally and is not introduced systemically. This is important since glaucoma medications can be active on the heart as well and can have unintended effects when dosed 
systemically. The effective dose can also be much higher than when dosed in the form of eye drops. A recent example of this has been shown in the elution of Poly(vinyl alcohol) from a contact lens. This is in effect a time release of a moisturizing agent from the contact lens. Poly(vinyl alcohol) is one of the standard actives in a moisturizing eye drop.

While a contact lens can release active molecules, it can also collect molecules from the tear fluid. In this way the contact lens can collect important molecules from the tear fluid for diagnostic purposes. The tear levels of a molecule used for diagnostic purposes needs to be correlated with normal and abnormal levels in blood. Then the lens can be analyzed with respect to concentration for purposes of the diagnosis. The lens will exchange saline with the tears and partition the active that one is looking at according to a thermodynamic equilibrium partition. There are many interesting molecules that are known to be in tears: glucose, hormones, markers for VEGF expression etc.

\section{Intraocular Lenses (IOL):}

2.1. EarlyHistory [12]: These synthetic lenses (IOLs or intraocular lenses) are inserted into the capsular bag to replace the natural cataractous lens that is removed during surgery. This is a very different application than contact lenses and as such has a very different profile of engineering properties which must be achieved.

This device is much more recent than that of contact lenses and has a much smaller total market size. Being a bodily implant it is also subject to much more scrutiny than contact lenses, which could be described as easily retrievable implants. Consider that if there is an adverse event, the lens must be explanted by a second surgical procedure. If there is an adverse event with a contact lens, one can usually just stop wearing the lenses and administer some medicinal eye drops. The stakes are much higher for an IOL. The FDA guidance requires stability testing for a 20 year implantation life. Ten million cataract operations are conducted annually in the US and Europe alone, making it the most common surgical procedure. It is thought that given longer life expectancy that almost everyone will suffer cataracts sooner or later. One of the causes of cataract formation is thought to be connected with cumulative UV exposure.

The materials history of the IOL starts with Poly(methylmethacrylate), PMMA. In 1940, Royal Air Force Flight Lieutenant Gordon Cleaver was wounded in a dog fight during the battle of Britain. Bullets shattered the canopy of Cleaver's plane which was made of PMMA. Tiny shards of the PMMA were imbedded into Cleaver's eyes. He eventually parachuted to safety. Ophthalmologists were unable to remove the shards. Sir Harold Ridley was one of his ophthalmologists. They could only wait and see what happened. As the years followed an extraordinary thing happened: nothing. Such was the realization that PMMA could be a useful material for ocular implantation. Evidently it was biocompatible and was inert in the environment of the eye.

In the late 1940's cataract surgery was already being performed. Once the natural lens was removed, patients became very far sighted and very high diopter lenses were required for the patient to regain normal vision, i.e. those euphemistically referred to as "coke bottle glasses." Ridley persuaded the London based Rayner Optical Company to manufacture an IOL using the same PMMA material that was imbedded in Cleaver's eye. On November 29, 1949, Ridley performed a cataract surgery and inserted the artificial lens in the patient's eye. Ridley announced his findings to the world in July of 1951 at the Oxford Ophthalmological Congress and was greeted by hostility and brandished as an outlaw. This was in part due to jealousy but also in part because of the lack of informed consent procedures and ethical review boards in those days. Entrenched technology gurus of the time summarily dismissed and rejected a new thought and brilliant discovery that would 
eventually come to impact the daily lives of tens of millions of individuals. This is an exemplification of the phenomenon of vehement peer rejection at work which can impede the acceptance of revolutionary disruptive technology. By the 1970's the criticism began to ebb and on February 9, 2000 Ridley was knighted by Queen Elizabeth II.

2.2. PMMA IOL: While these lenses represented a huge step forward in the ability to restore sight to post cataract patients, they were only the initial version and suffered a severe shortcoming: the lens required a 5-7 mm incision. Such a large incision in the eye requires sutures, is prone to infection, and prolongs the recovery time. PMMA IOL's are still used in Asia and Africa where the extra expense of the improved foldable lenses cannot be easily tolerated. An interesting characteristic of PMMA is that it cannot be heat sterilized because of its low glass transition temperature, Tg. Thus other rigid materials such as polycarbonate and polysulfone have been considered because of their autoclavability.

2.3. Soft Foldable IOL: Foldable IOLs are made from silicone and acrylic elastomers and are designed to be folded for insertion through a much smaller incision than that used for rigid, nonfoldable PMMA IOLs. Silicone elastomers were the basis of the original foldable IOLs due to optical clarity and the wide and successful use of solid crosslinked silicone elastomer in a variety of implants in other parts of the body. Poly(dimethyl siloxane) copolymerized with diphenyl siloxane is used in high refractive index foldable IOLs. The acrylic lenses can be either hydrophobic or hydrophilic and there is currently some discussion about the merits of each. It appears that the hydrophilic lenses have some predisposition to calcification or opacification. This is still under review at this writing. The soft lenses can be tightly rolled up and placed into an inserting tool for insertion. The goal is to have an incision less than $3 \mathrm{~mm}$ for insertion. This is in effect the solution for the problems mentioned above for the PMMA IOL. A modern IOL with the haptics is shown in Figure 2.

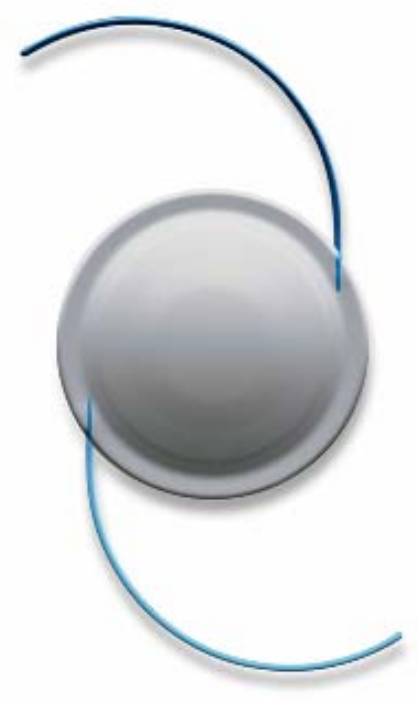

Figure 2. A picture of a modern IOL: Tecnis Multifocal by Advanced Medical Optics, Inc. The haptics on the side are for securing it in the capsular bag.

2.4. Accommodating IOLs [13]: Standard IOLs do not have the ability to provide accommodation for near and far vision, i.e. multifocal capability for addressing presbyopia. Note that only the multifocal is shown above in the figure. In recent years there has been 
considerable activity in the development of accommodating IOLs through advanced mechanical, geometric and optical designs coupled with modifications of existing IOL materials, primarily silicones and hydrophilic acrylics. In this case, the lens is essentially a very soft flexible lens that changes its curvature distance relative to the retina by using the natural muscular action exerted on the capsular bag to provide the needed accommodation. The accommodation of the lens requires special materials with particular properties in order to achieve the desired movement. The first accommodating IOL approved by the FDA was the Crystalens Model AT-45 in 2003 by Eyeonics Inc. The lens is a modified hinged, platehaptic silicone lens with polyimide loops. Polyimide is known to produce fibrosis which locks the haptics in place to stabilize the optical axis. Another accommodating IOL based on silicone material is the Synchrony dual-optic device by Visiogen Inc. which is not as yet commercialized.

2.5. Injectable IOL [14]: The Helmholtz theory of presbyobia assumes that the natural lens in the eye becomes less flexible with age and that the muscles are unable to bring enough force to bear to move it. A natural thought is that accommodation could be restored by replacing the stiff presbyobic lens with a material mimicking the young crystalline lens. Such a material must be soft, transparent and have a refractive index close to that of the natural lens. There must be a surgical procedure that allows for the extraction of the crystalline lens while preserving the capsular bag. A prepolymer can then be injected into the bag for in situ polymerization into the bag which serves as a mold to impart the correct power after the completion of the polymerization.

2.6. UV and Visible Light Filtration [15]: The natural lens which is being removed because of the cataracts has UV filtering properties. The materials for the lenses discussed above are transparent to the UV. Placing one of these into the capsular bag in its natural condition would expose the retina and other light sensitive elements in the back of the eye to damaging UV radiation. To filter UV one has the standard molecules such as benzophenones and benzotriazoles which can be incorporated by admixing into the polymers. Benzophenones are mostly efficacious in the UVB while Benzotriazoles also filter in the UVA region. While the potential damage from UVB is clear, clinical researchers in more recent times have become focused on the potential danger from chronic exposure to UVA, as well. Since the FDA guidance requires a 20 year useful implantation lifetime, one needs to carefully test for photo degradation and leaching using accelerated testing. For the PMMA hard lenses there is little danger of leaching. For the soft lenses that risk goes up and the useful lifetime must be empirically determined by accelerated aging studies. For the hydrophilic lenses, the presence of the water increases the leaching risk. In such case that the leaching becomes an issue, one must employ filters that are functionalized with double bonds so that they can be co-polymerized with the basic lens polymer.

In recent years, visible light blocking IOL's have been introduced. These are commonly referred to as blue light-blocking IOL's where blue generally refers to the broad range of visible light between 400 and $500 \mathrm{~nm}$. These IOL's use yellow dyes and are thought to protect retinal pigment epithelial cells from blue-light damage or photo-toxicity. The artist Claude Monet actually insisted on wearing yellow-tinted spectacles to recover or improve his color perception after having undergone cataract surgery. While there is little doubt that blue-blocking IOL's have some benefit, there is still some debate and uncertainty as to the magnitude of this benefit regarding: visual performance, circadian rhythms, and alleviation of the risk of age-related macular degeneration.

2. Intracorneal Rings [16] : In the early 1980's J. Petricciani, J. Krezanowski, G. Reynolds and J. Flemming did some pioneering work. The concept was to reshape the cornea and potentially correct common vision problems such as myopia by mechanically altering the 
cornea's curvature. This could be achieved by inserting precision-engineered polymers in the periphery of the cornea, but outside the central optical zone which is the critical area for clear vision.

The device referred to as an intracorneal ring or intrastromal corneal ring, ICR, is placed in the plane of the eye a few hundred microns below the surface into channels created for that purpose. There are generally two rings inserted, each of which spans an arc of 150 degrees. The perimeter of each ring is about $7 \mathrm{~mm}$. The diameter of the rings can vary from about $.25 \mathrm{~mm}-.45 \mathrm{~mm}$. The cross sectional profile is the result of proprietary engineering. Increased diameter of the rings leads to greater degrees of central corneal flattening. About 4 diopters of myopic correction can be obtained with the $.45 \mathrm{~mm}$ ring. The use of the device has the benefit of removability as compared to laser ablative surgery. The risk of infection is small due to the small incision.

The material used for this device was and is poly-methylmethacrylate. Its successful utilization within the IOL sector made it an obvious choice. The mechanism for obtaining the corneal flattening has been studied using finite element calculations using the modulus of the soft cornea and the rigid PMMA ICR. One creates in essence a reinforcement of the soft corneal matrix with the high modulus PMMA ring. The intrinsic inertness and high modulus properties of PMMA appear to make it ideal for this application. No materials developments are anticipated. The device is known as Intacs and was launched by Keravision, Inc. It was approved for use in the USA in 1999.

The Technology passed to Addition Technology, Inc. in 2001. It was subsequently shown that the device could be very effective in the treatment of keratoconus, a somewhat rare condition affecting 1/2000 of the population. This is a condition during which the cornea bulges leading to a severe loss of vision and eventual blindness. While rigid contact lenses can be an intermediate treatment, the long term prognosis can be a corneal transplant. With the ICR's ability to reinforce and flatten the weakened cornea, as noted above, considerable improvement can be obtained for patients combating this disease. Figures $3 \mathrm{a}$ and $3 \mathrm{~b}$ show a pair of free standing rings and a human eye containing the implants, respectively.

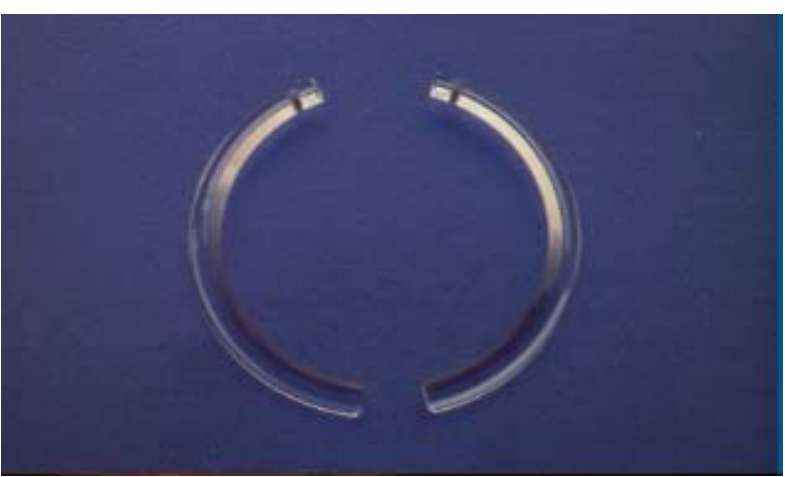

Figure 3a. A matched pair of Intacs ICR's from Addition Technology, Inc.

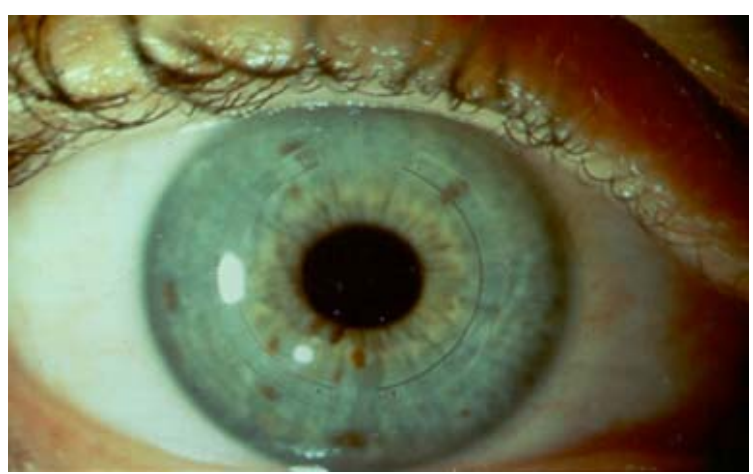

Figure 3b. A pair of Intacs implanted in a human eye.

4. Viscoelastic Agents [17] play an important role in ophthalmic surgery as soft surgical tools used to protect the corneal endothelium during cataract extraction, manipulation of capsular tissue and the IOL, in glaucoma surgery and to facilitate vitreoretinal surgery. They are known as Ophthalmic Viscoelastic Devices, OVD's. Corneal endothelial cell damage was until recently a common occurrence during IOL insertion and removal by the inadvertent rubbing of surgical instruments. To prevent this, OVD's have been introduced into the eye to protect the corneal endothelium while allowing the surgeon to safely manipulate the implants with surgical instruments. This single layer endothelium has no regenerative capacity. 
Hyaluronic acid was isolated from the vitreous humor in 1934 by Mayer and Palmer. Subsequent research focused on the development of artificial vitreous bodies. Other materials used for this purpose are hydroxypropylmethylcellulose, chondroitin sulphate, collagen and various mixtures of these materials. These other materials on their own did not have sufficient viscoelastic properties for many applications and sodium hyaluronate has become the most widely used material. The hyaluroinic acid is extracted from rooster combs or produced by bacterial fermentation and subsequently subjected to extensive purification. A key innovation in this area has been the development of cohesive and dispersive OVD's. Cohesive OVD's have high molecular weight, in excess of 2.5 million Daltons and demonstrate very high viscosity at zero shear rate and lower viscosity at finite shear rates. They are highly pseudoplastic showing Bingham fluid like behavior. Dispersive OVD's having molecular weights less than 1 million Daltons do not exhibit high cohesion and thus disperse, flow, throughout the chamber and can adhere to tissue. The actual OVD or molecular weight employed depends on the particular surgical application and the need for viscoelastic properties. The dispersive OVD is harder to remove because it has liquid like properties, while the cohesive is easier to remove since it behaves as a solid gel during the removal process. Healon ${ }^{\circledR} 5$ by Advanced Medical Optics, Inc. is a viscoelastic OVD having both cohesive and dispersive properties.

Its use for ophthalmology was patented in the USA in 1979 with the first launch of Healon ${ }^{\circledR}$ in 1980 . Currently it has been suggested as a miracle nutrition supplement to address a wide range of conditions from arthritis to skin aging!

5. Future Developments in Materials for Ophthalmology: As this is being written it is an unprecedented time for innovation in the area of medical devices. What are some of the things that can be expected?

- Better surface treatments for biocompatibilizing otherwise useful device materials

- Functional surface or bulk treatments for self disinfecting contact lenses.

- Drug releasing contact lens systems or inserts for the efficacious delivery of therapeutics for the eye.

- Devices with short term drug release to counter the effects of post operative trauma.

- Contact lens systems for use in tear diagnostics.

- An IOL that truly restores a full range of accommodation without resulting in post-capsular opacification.

6. The Innovation Process [18] : Most of the work leading to the innovations described above has occurred within the domain of the private sector with only marginal governmental support. To the extent the government is involved there will be motivations of advancing the overall scientific standard. For the private sector, developmental costs will be looked upon as investments with the intent of generating a return. For a new innovative product one can perform economic modeling calculations to consider the following factors: total market size being addressed by the new product; growth of market; resources required to develop, manufacture and market the product; duration of development; selling price of the product; competition; intellectual property; regulatory strategy; risk of failure etc. It becomes apparent that small markets will not permit large developmental investments, while larger markets will. To some degree of approximation, Innovation will therefore be proportional to the ratio Market Size/ Product Development Cost. The Contact Lens and IOL markets are of the order of billions of dollars and hence the attraction of being a player in those markets attracts considerable development investments because of the commensurate returns. In fact, within ophthalmology that is where most of the materials innovations have taken place. The IOL Market is smaller than the Contact Lens Market while having higher product development 
costs due to the surgical implant nature of the device and hence the materials innovations for this class of product lag that for Contact Lenses. The Intracorneal Ring Market is very small in comparison. Thus given the large clinical development expenses for a surgical implant of this type one would not reasonably expect large investments in new ICR materials even though the resulting devices might be more efficacious. One would therefore expect the relative Materials Innovation to be Contact Lenses> IOL's> ICR's. Reflecting retrospectively on the review presented in the foregoing, that is exactly what one observes!

Acknowledgements: The authors would like to thank Dr. Arlene Gwon for her careful reading of the manuscript and constructive comments. The authors would also like to thank Nancy and Michael Connelly for formatting the manuscript. The authors also would like to thank J.P. Dismukes, M. Lowery and B. Lundgren for contributing useful additions to the paper.

\section{References}

[1] D. Fonn, D. Sweeney, B.A. Holden and D. Cavanagh, Eye and Contact Lenses 31(1), pp. 23-27, (2005); S.M. Dillehay, Eye and Contact Lenses, 33(3), pp. 148-55, (2007).

[2] European Patent Application 0580435 A1; J. Watanabe, Encyclopedia of Biomaterials and Biomedical Engineering, Marcel Dekker, Inc., 2004, p. 790 ff; T. Goda and K. Ishihara, Expert Rev. Med. Devices 3:2,167 (2006).

[3] US Patents: 5,583,163; 6,407,145; 5,508,317; 5,789,464; 5,849,810; A. Muhlebach, B.Muller, C. Pharisa, M. Hofmann, B. Seiferling and D. Guerry, J. Polym. Sci.: A: Chemistry, $\underline{35}, 3603$ (1997).

[4] G. R. Bell, Contact Lens Spectrum, p. 31ff, November 1998.

[5] E. Bennett, Review of Cornea \& Contact Lenses, p. 15, May 2007.

[6] P.C. Nicolson and J. Vogt, Biomaterials, 22, 3273(2001); P.C. Nicolson and J. Vogt, Contact Lenses: Silicone Hydrogels in Encyclopedia of Biomaterials and Biomedical Engineering, Informa Healthcare, 2006, 1, p 1 ff ; D. Sweeney, Silicone Hydrogels: The Rebirth of Continuous Wear Contact Lenses, Butterworth-Heinemann, (2000 first edition/2004 second edition); J. Kunzler and R. Ozark, J. Applied Polym. Sci., 65:6,1081 (1997); L. Alvord, J. Court, T. Davis, C.F. Morgan, K. Schindhelm, J. Vogt and L. Winterton, Optometry and Vision Science, 75:1, 30 (1998); B.J. Tighe, Contact Lens Materials in A. Phillips and L. Speedwell, Contact Lenses, Butterworth-Heinemann, 2006, pp59-78.

[7] US Patent Applications:20060063852; 20060004165; 20060001184; 20060142410; US Patents: 6,867,245; 6,951,894; 5,965,631; 5,849,811; 5,789,461; 5,776,999; $5,760,100 ; 5,849,811 ; 5,070,215 ; 5,610,252 ; 6,822,016$.

[8] US Patent 6,244,707

[9] US Patents: 5,272,010; 4,553,975; 4,559,059; 5,292,350; 6,149,842.

[10] D.F. Sweeney, A. Vannas, T.C. Hughes, M.D.M. Evans, K.M. McClean, R.Z. Xie, V.K. Pravin and R.K.Prakasam, Clin. Exp. Optom., 91:1, 56(2008); M.D.M. Evans, K.M. McLean, T.C. Hughes, and D.F. Sweeney, Biomaterials, 22, 3319 (2001).

[11] L.C. Winterton, J.M. Lally, K.B. Sentell and L.L. Chapoy, J. of Biomedical Materials Research, Part B: Applied Biomaterials, $\underline{\text { 80B }}$, 424 (2006); Encyclopedia of Biomaterials and Biomedical Engineering, 2004, p.1105ff.

[12] Sir Harold Ridley and His Fight for Sight, David, J. Apple, Slack Incorporated, 2006.

[13] Cataract and Refractive Surgery Today, May 2004, p. 16.; US Patents: 6,849,091; $5,476,514 ; 6,387,126 ; 7,048,760 ; 6,884,261 ; 5,476,514 ; \mathrm{R}$. Menapace, O. Findl, 
K.Kreichbaum and Ch. Leydolt-Koeppl, Graefe's Clin. Exp. Ophthalmol, 245, 473(2007); H.B. Dick, Current Opinions in Ophthalmology, 16:8, 26(2005).

[14] Refractive Lens Surgery, Springer, 2005, S. Norrby, p. $173 \mathrm{ff}$.

[15] L. Werner, R.J.Olson and N. Mamalis, Ophthalmol. Clin. N. Am., 19, 469(2006); M.A. Mainster, Arch. Ophthalmol., 123, 550(2005).

[16] US Patent 4,452,235; T. Chirila and C.R. Hicks, Corneal Implants in Encyclopedia of Biomaterials and Biomedical Engineering, Marcel Dekker, 2006, p. $392 \mathrm{ff}$.

[16] T.J. Liesegang, Viscoelastic substances in Ophthalmology, Surv. Ophthalmol, $\underline{34}$, 268 (1990); K.L. Gos and B. Benfield, Drugs, 47, 536 (1994).

[17] R. G. Cooper, Winning at New Products, Perseus Publishing, Cambridge, Ma, 2001; T. Kuhn, The Structure of Scientific Revolutions, University of Chicago Press, 1996.

General References below are indicated for the major subsections in the text. The interested reader can go there to access the technical details which are not covered in this review which focuses on the innovation aspect. More specific references are given by the numbered references in the text and listed above:

\section{Contact Lenses}

- Contact Lens Spectrum, July 1987.

- Hydrogels, Encyclopedia of Biomaterials and Biomedical Engineering, MarcelDekker p. 790ff, (2004).

- Contact Lenses, Kirk-Othmer Encyclopedia of Chemical Technology, Fourth Edition, Volume 7, John Wiley \&Sons.

- Szczotka-Flynn, Contact Lens Spectrum, June, 2006, p. 23.

- D. Fonn, Contact Lens Spectrum, June, 2006, p. 48.

- J.E. Key, Eye \& Contact Lens, 33(6), 343 (2007).

- P.C. Nicolson, and J. Vogt, Biomaterials, 22, 3273 (2001).

- R.F. Heitz, The History of Ophthalmology, 11-3A, Kugler Publications, (2003).

\section{Surgical Implants}

- A.W. Lloyd, R.G.A. Faragher and S.P. Denyer, Biomaterials, 22, 769 (2001).

- D.L. Wise, Encyclopedic Handbook of Biomaterials and Bioengineering, Part B: Applications, 2, Marcel Dekker, (1995): Biomaterials Used for Intraocular Lenses, F.R. Christ, Y. Buchen, J. Deacon, C.M. Cunanan, J.E. Giamporcaro, P.M. Knight, J.I. Weinschenk, and S. Yank, pp. 1261-1313.

- M. Chehade and M.J. Elder, Australian and New Zealand Journal of Ophthalmology, 25, 255 (1997).

- D.F. Sweeney, Optometry and Vision Science, 83:3, 133 (2006).

- M. Tehrani, H.B. Dick, B. Wolters and T. Pakula, Ophthalmologica, 218, 57 (2004).

- R.J. Olson, (Panel Moderator), Supplement to Cataract \& Refractive Surgery Today Europe, January/February, (2006).

- P. White, Contact Lens Spectrum, February, 2007, p. 42.

- W. Bethke, Review of Ophthalmology, November, 2007, p. 67.

- S.S. Lee, P. Yuan and M.R. Robinson, Ocular Implants for Drug Delivery in Encyclopedia of Biomaterials and Biomedical Engineering, Marcel-Dekker, 2004, pp 1105-1119. 
- Y. Ali, K. Lehmussaari, Industrial Perspective in Ocular Drug Delivery, Advanced Drug Delivery Reviews, 58, 1258 (2006).

- T.V. Chirila and C.R. Hicks, Corneal Implants in Encyclopedia of Biomaterials and Biomedical Engineering, Marcel-Dekker, 2004, pp 392-398; J.B. Leach and C.E. Leach, Hyaluronan in Encyclopedia of Biomaterials and Biomedical Engineering, Marcel-Dekker, 2004, pp779-789.

- L. Germain, C.J. Giasson, P. Carrier, S.L. Guerin, C. Salesse and F.A. Auger, Tissue Engineering of the Cornea in Encyclopedia of Biomaterials and Biomedical Engineering, Marcel-Dekker, 2004, pp 1534-1544.

- J.L. Bourges, C. Bloquel, A. Thomas, F. Froussart, A. Bochot, F. Azan, R. Gurny, D. BenEzra and F. Behar-Cohen, Intraocular Implants for Extended Drug Delivery Therapeutic Applications, Advanced Drug Delivery Reviews, $\underline{58}$, 1182(2006).

- K.T. Doan, R.J. Olson and N. Mamalis, Current Opinions in Ophthalmology, $\underline{13: 1, \mathrm{Feb}}, 24(2002)$. 